\title{
ANALYTICAL APPROACH TO AUGMENTING SITE PHOTOS WITH 3D AS-BUILT BORED PILE MODELS
}

\author{
Fei Dai \\ Ming Lu \\ Dept. of Civil and Structural Engineering \\ Hong Kong Polytechnic University \\ Hong Kong, CHINA
}

\begin{abstract}
To acquire information of the invisible underground infrastructure, non-destructive subsurface imaging technologies are generally utilized. In construction, the recorded data of as-built pile excavation is archived in Koden test result sheets. The test result is in the form of literal document and identified by the pile numbering. The graphical presentation of the test result may provide a clearer and easier way for engineers to investigate the pile alignment by merging the result onto the real site photo. The key is to devise a "virtual camera" to project the three-dimensional as-built model of the bored pile onto a twodimensional view of the site scene, resulting in an augmented photo of the bored pile. The mathematical equations are formulated to allow for parameterization of the projection. A case of augmenting a ground site photo with as-built underground data for the purpose of investigation of alignment for a bored pile construction is demonstrated.
\end{abstract}

\section{INTRODUCTION}

In constructing foundations of a structure, the current practice resorts to the as-built records in the form of site photos annotated with detected underground information for quality assurance and defect investigation. At construction sites, site engineers routinely take site photos of an on-going project to keep timely records for documentation. Recent advances in electrooptical technologies have empowered the off-the-shelf digital cameras to obtain high quality images, while maintaining portability and convenience of picture taking. With site photos, a particular state of the site situation, including building products, construction resources and site layout, can be easily captured and kept (Dai and Lu 2008).

Utilizing photos and photography techniques to cater to the needs of problem solving has been applied in a variety of disciplines. In regard to applications in construction engineering and project management, Kim and Kano (2008) investigated the actual site progress by contrasting the photo-generated 3D site models to the as-planed VR scenes. Waugh (2006) employed construction site photos to implement a panorama view of the site situation for better site supervision. To preserve evidence against potential construction-caused damage claims, the site photos have been analytically processed by contractors to measure 3D geometries of buildings adjacent to the construction site (Luhmann and Tecklenburg 2001). In addition, Abeid and Arditi (2002a, 2002b) advanced a time-lapse photography technique for project management by recording activities of a construction site with a series of photographs, enabling the playback at optional frame rates. With regard to photo applications in construction, however, the following two questions are yet to be clarified: (1) the photographing process entails projecting spatial rays of light onto the photosensitive film, but the underlying analytics has yet to be enlightened to construction professionals; and (2) the research effort strives for extracting data from two dimensional images and mapping them into a three dimensional object space, but the reversing process of immersing $3 \mathrm{D}$ as-built models into a photo has yet to be attempted in construction research.

Underground construction products such as bored piles features invisibility and complexity, leading to difficulty for site investigators to check the quality of the underground product being built over time and monitor the construction progress. To address this difficulty, some subsurface imaging technologies (e.g. ultrasonic waves, ground penetrating radars) have been employed for detecting the features of the underground infrastructure. Wave signals are reflected from underground and further processed to yield information about the distribution of the physical properties in relation to conditions of the underground product. Interpretation of the processed data can indicate the position of the underground target object (Jeong and Abraham 2004). This practical need and the technical methods available spawn the present research effort to develop an analytical approach to augmenting site photos with the three-dimensional as-built data from subsurface imaging technologies so as to exhibit a richer and more integral view of the construction site situation. 


\section{Dai and $\mathrm{Lu}$}

The remainder of this paper first briefly introduces the Koden test method which is adopted as the current practice for outlining the profile of bored pile excavation. Then, this paper makes an attempt at establishing photo-augmenting equations by which the $3 \mathrm{D}$ as-built models of bored piles can be analytically immersed onto the $2 \mathrm{D}$ site photos. Last, a case to adding real underground data onto a ground site photo for the purpose of investigation of bored pile construction quality is presented.

\section{MODELING 3D AS-BUILT BORED PILES}

To acquire geometric data of the invisible underground infrastructure, non-destructive subsurface imaging technologies are generally employed in construction, such as electromagnetic methods, magnetic methods and acoustic emission methods (Jeong and Abraham 2004).

The inspection technique of Koden test is to take the acoustic emission method, of which the basic principle is to make use of ultrasonic waves to measure the dimensions of the shaft and bellout of a just excavated bored pile. The testing instruments consist of a four-direction ultrasonic wave sensor, a winch system to lower and lift the sensor within the pile hole, and a system controller for outputting and recording the measurement results.

A four-direction sensor can take measurements in two orthogonal directions which are defined as XX'-axis and YY'-axis respectively (Figure 1a). As the sensor is lowered or lifted inside the excavation hole, ultrasonic waves are emitted and propagated through the filling or supporting fluid (i.e. water, bentonite). As the emitted ultrasonic wave reaching the wall surface, it is reflected back and received by the sensor. By determining the duration of the ultrasonic wave propagation, the conditions of sidewalls in the bored pile hole can be obtained and directly printed in the recording paper. As to the output format, the recording paper contains the verticality of sidewalls, hole size, bellout size and depth of excavation (Figure 1b), and based on this information, a three-dimensional, as-built model of the bored pile can be quickly sketched.

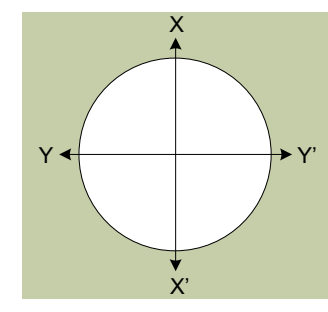

a: Plan view of the bored pile

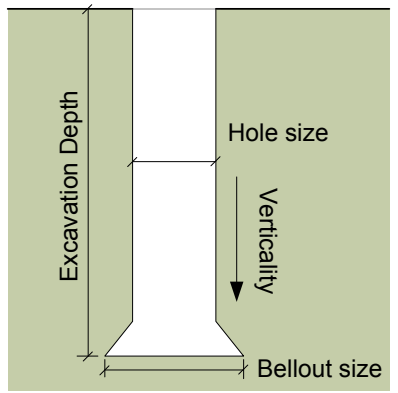

b: Elevation view of the bored pile

Figure 1: Orthographic projection views of the bored pile

In practice, the recorded information for the as-built pile excavation is archived in the form of the Koden test result sheet. The result is in the form of literal document and identified by the pile numbering. The graphic presentation of the result may provide a clearer and easier way for engineers to investigate the as-built pile by merging the result onto the real site photos. The key link is to use a "virtual camera" to project the three-dimensional as-built model of the bored pile onto a twodimensional view of scene about the site, resulting in an augmented photo of the bored pile. The following section will discuss the implementation of the photo-augmentation equations in detail.

\section{PHOTO-AUGMENTATION EQUATIONS}

The photo-augmentation equations are related to the imaging mechanism in the optical science. The imaging mechanism is concerned with the process that the reality in the three dimensional space is projected onto the flat film of the camera, generating a view of a three dimensional scene at a particular time. The core of this process is to establish a linkage between the position of the point in the three dimensional space with the position of the same point on the camera image plane (film). The following section interprets the camera imaging mechanism, based on which, the mathematical equations are formulated to allow for parameterization of projecting a three dimensional model onto a related two dimensional photo.

\subsection{Camera Imaging Mechanism}

To take a photo, we need first to position the camera at a particular location in the space. Then we need to decide the camera's orientation: in which direction we point the camera and how should we rotate it around the line of sight? Finally, we 


\section{Dai and $L u$}

snap the shutter, the scene is tailored to the size of the aperture of the camera, and light from the visible surfaces is projected onto the image plane of the camera. Figure 2 gives an illustration of a camera's position and orientation when photographing an object.
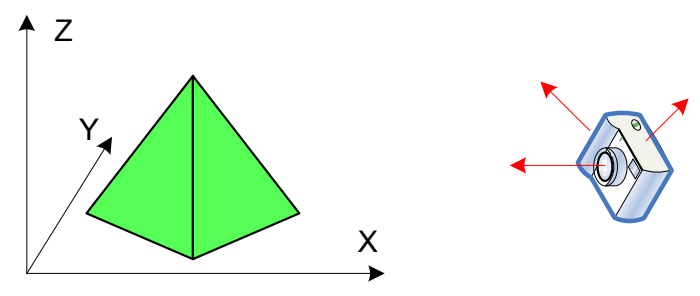

Figure 2: A camera position and orientation when photographing a scene

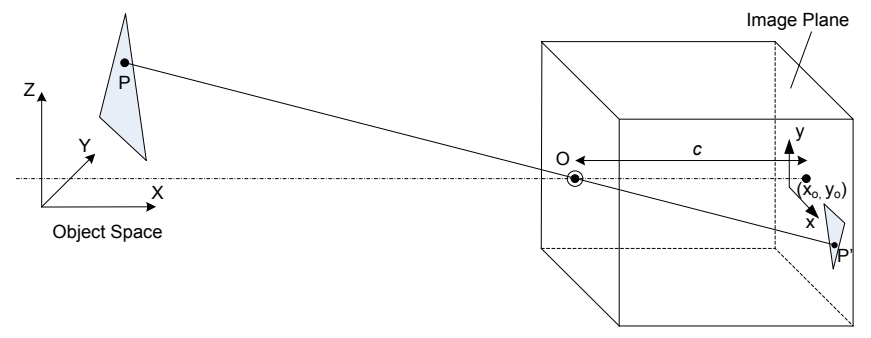

Figure 3: Camera image forming mechanism

We then attempt to establish the mathematical relationship between the point positions in the object space and in the image plane of the camera. The coordinate system in the object space is defined as the object coordinate system, and the one in the image plane is defined as the image coordinate system. Both coordinate systems are right-handed Cartesian. Figure 3 shows the camera image forming mechanism. The lens of the camera is modeled by a single point $O$, known as the perspective center and its location in object space is $\left(X_{o}, Y_{o}, Z_{o}\right)$. The principal distance ( $c$ in Figure 3 ) refers to the perpendicular distance from the perspective center $O$ to the image plane and the principal point $\left(x_{o}, y_{o}\right.$ in Figure 3$)$ is where the line, which is through the perspective center and perpendicular to the image plane, intersects the image plane. The orientation of the camera is characterized by three Euler orientation angles. In ideal situations, the object point, the perspective center, and the image point are aligned along a straight line $\left(P, O, P^{\prime}\right.$ as shown in Figure 3$)$. This yields the collinearity condition in which the vector from the perspective center to the image point aligns with the vector from the perspective center to the object point

$$
\left[\begin{array}{c}
x_{n}-x_{o} \\
y_{n}-y_{o} \\
-c
\end{array}\right]=\lambda \boldsymbol{M}\left[\begin{array}{c}
X_{n}-X_{o} \\
Y_{n}-Y_{o} \\
Z_{n}-Z_{o}
\end{array}\right] .
$$

In (1), $\boldsymbol{M}$ is the $3 \times 3$ rotation matrix, $\lambda$ is the scale factor, and $\boldsymbol{p}_{\boldsymbol{n}}=\left(x_{n}, y_{n}\right)^{T}$ and $\boldsymbol{P}_{\boldsymbol{n}}=\left(X_{n}, Y_{n}, Z_{n}\right)^{T}$ are the coordinates of the $n$th target point in the image plane and the object space, respectively. Assume that $m_{i j}(i, j=1,2,3)$ are the elements of the rotation matrix $\boldsymbol{M}$, algebraic manipulation of (1) yields the Collinearity Equations, which relates the $n$th target point in the object space to the corresponding point in the image plane (Wong 1980; Wolf 1983; McGlone 1989), as in

$$
\begin{aligned}
& x_{n}=x_{o}-c \frac{m_{11}\left(X_{n}-X_{o}\right)+m_{12}\left(Y_{n}-Y_{o}\right)+m_{13}\left(Z_{n}-Z_{o}\right)}{m_{31}\left(X_{n}-X_{o}\right)+m_{32}\left(Y_{n}-Y_{o}\right)+m_{33}\left(Z_{n}-Z_{o}\right)}, \\
& y_{n}=y_{o}-c \frac{m_{21}\left(X_{n}-X_{o}\right)+m_{22}\left(Y_{n}-Y_{o}\right)+m_{23}\left(Z_{n}-Z_{o}\right)}{m_{31}\left(X_{n}-X_{o}\right)+m_{32}\left(Y_{n}-Y_{o}\right)+m_{33}\left(Z_{n}-Z_{o}\right)} .
\end{aligned}
$$

By (2), the $X, Y, Z$ coordinates of the object point in the object space can be transformed into the $x, y$ coordinates of the corresponding image point in the image plane. 


\section{Dai and $L u$}

\subsection{Rotation Matrix Determination}

In this paper, we introduce the rotation angles azimuth $(\alpha)$, tilt $(t)$, and swing $(s)$ for determining the rotation matrix $\boldsymbol{M}$, by which the orientations of the camera in the object space are defined. Figure 4 illustrates the camera image plane and the three angular orientations - azimuth, tilt, and swing. In Figure 4, the point $O$ is the perspective center of the camera, and $o$ is the principal point on the image plane. Projecting $O$ onto the XY-plane of the object coordinate system yields the point $P_{o}$, $O o$ is the line perpendicular to the image plane, and the extension of the line $O o$ intersects the XY-plane yielding the point $P_{d}$. By plotting an auxiliary $\mathrm{Y}^{\prime}$-axis passing through $P_{o}$ on the XY-plane, azimuth $(\alpha)$ is defined as the clockwise angle measured from the $\mathrm{Y}^{\prime}$-axis to the line $P_{o} P_{d}$. Tilt $(t)$ is the angle between the vertical line $O P_{o}$ and the camera optical axis $O P_{d}$. The vertical line $O P_{o}$ intersecting the image plane yields the point $n . n$ is the photographic nadir point. Swing ( $s$ ) is defined as the clockwise angle measured on the image plane from the positive vertical axis y downward the line emanating from the principal point $o$ to the photographic nadir point $n$. Actually, the line on lies in the vertical plane that is parallel to the YZ-plane and intersects the image plane at the principal point $o$.

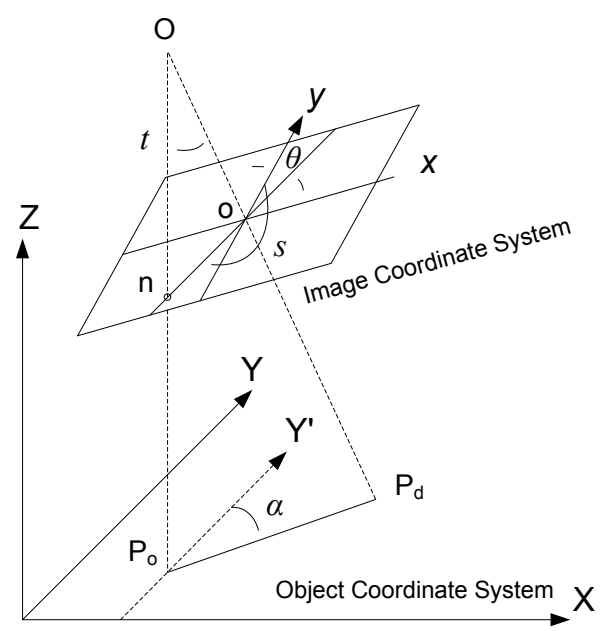

Figure 4: The camera image plane showing the three angular orientations - azimuth, tilt, and swing

For the rotation matrix $\boldsymbol{M}$, the elements $m_{i j}$ are functions of the rotation angles $(\alpha, t, s)$. By use of $(\alpha, t, s)$, the image coordinate system can be rotated parallel to the object coordinate system. To develop the rotation formulas, we assume that the object coordinate system and the image coordinate system are both moved to the perspective center $O$ of the camera, denoted by the XYZ-coordinate system and the xyz-coordinate system respectively as shown in Figure 5a. The rotation formulas can be developed sequentially in three independent steps. In each step, we rotate one axis and change the positions of the other two axes (Figure 5b-d). Now, we rotate back the XYZ-coordinate system to the xyz-coordinate system to determine the rotation formulas.

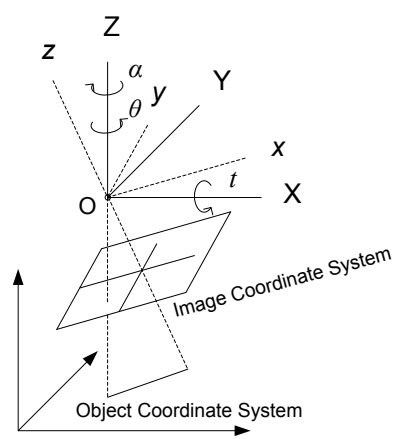

a: Rations in azimuth, tilt, and swing

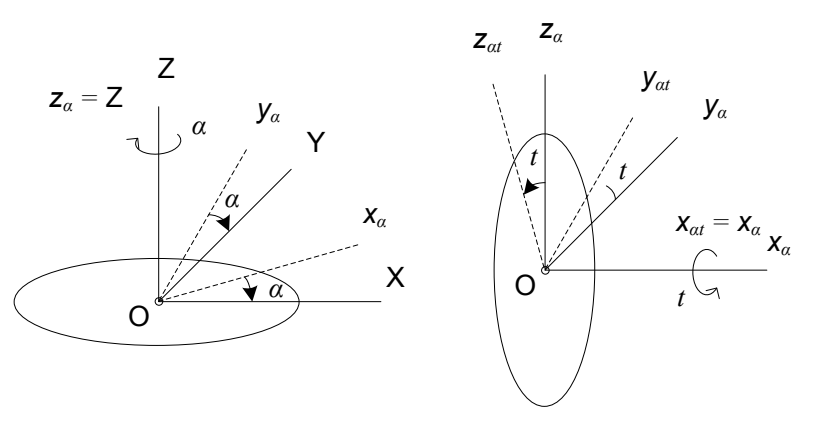

b: First rotation in azimuth

c: Second rotation in tilt

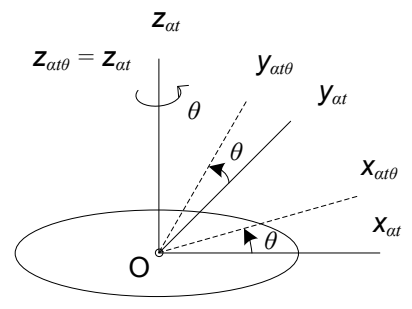

Figure 5: Rotations of azimuth, tilt, and swing in the three-dimensional space 


\section{Dai and Lu}

Step 1 is about the first rotation in azimuth $(\alpha)$, in which the XYZ-coordinate system rotates a clockwise angle $\alpha$ about the Z-axis to generate an $\mathrm{x}_{\alpha} \mathrm{y}_{\alpha} \mathrm{z}_{\alpha}$-coordinate system (Figure 5b). The coordinates of any point in the XYZ-system can be calculated from the $\mathrm{x}_{\alpha} \mathrm{y}_{\alpha} \mathrm{z}_{\alpha}$-system by

$$
\boldsymbol{X}=\left(\begin{array}{ccc}
\cos \alpha & \sin \alpha & 0 \\
-\sin \alpha & \cos \alpha & 0 \\
0 & 0 & 1
\end{array}\right)\left(\begin{array}{l}
x_{\alpha} \\
y_{\alpha} \\
z_{\alpha}
\end{array}\right)=\boldsymbol{R}_{\alpha} \boldsymbol{x}_{\alpha} .
$$

Step 2 is about the second rotation in tilt $(t)$, in which the $\mathrm{x}_{\alpha} \mathrm{y}_{\alpha} \mathrm{z}_{\alpha}$-coordinate system rotates a counter-clockwise angle about the $\mathrm{x}_{\alpha}$-axis to generate an $\mathrm{x}_{\alpha \mathrm{t}} \mathrm{y}_{\alpha \mathrm{t}} \mathrm{z}_{\alpha \mathrm{t}}$-coordinate system (Figure $5 \mathrm{c}$ ). Since this time the rotation is counter-clockwise, we may simply add a minus before the angle to evaluate the rotation matrix. The coordinates of any point in the $\mathrm{x}_{\alpha} \mathrm{y}_{\alpha} \mathrm{z}_{\alpha}-\mathrm{system}$ from the $\mathrm{x}_{\alpha \mathrm{t}} \mathrm{y}_{\alpha \mathrm{t}} \mathrm{z}_{\alpha \mathrm{t}}$-system are

$$
\boldsymbol{x}_{\alpha}=\left(\begin{array}{ccc}
1 & 0 & 0 \\
0 & \cos t & -\sin t \\
0 & \sin t & \cos t
\end{array}\right)\left(\begin{array}{l}
x_{\alpha t} \\
y_{\alpha t} \\
z_{\alpha t}
\end{array}\right)=\boldsymbol{R}_{t} \boldsymbol{x}_{\alpha t} .
$$

In Step 3, the third rotation in swing is performed with regard to the angle $\theta$, which is defined by $\theta=s-180^{\circ}$ (Figure 4 ). The rotation of the angle $\theta$ is counter-clockwise about the $\mathrm{x}_{\alpha \mathrm{t}}$-axis, generating an $\mathrm{x}_{\alpha t \theta} \mathrm{y}_{\alpha t \theta} \mathrm{Z}_{\alpha t \theta}$-coordinate system which coincides with the xyz-system (Figure $5 \mathrm{~d}$ ). The coordinates of any point in the $\mathrm{x}_{\alpha \mathrm{t}} \mathrm{y}_{\alpha \mathrm{t}} \mathrm{z}_{\alpha \mathrm{t}}-$ system from the $\mathrm{x}_{\alpha t \theta} \mathrm{y}_{\alpha t \theta} \mathrm{z}_{\alpha t \theta}$-system can be calculated by

$$
\boldsymbol{x}_{\alpha t}=\left(\begin{array}{ccc}
\cos \theta & -\sin \theta & 0 \\
\sin \theta & \cos \theta & 0 \\
0 & 0 & 1
\end{array}\right)\left(\begin{array}{l}
x_{\alpha t \theta} \\
y_{\alpha t \theta} \\
z_{\alpha t \theta}
\end{array}\right)=\boldsymbol{R}_{\theta} \boldsymbol{x} .
$$

Substituting $\theta=s-180^{\circ}$ into (5a), we obtain

$$
\boldsymbol{x}_{\alpha t}=\left(\begin{array}{ccc}
-\cos s & \sin s & 0 \\
-\sin s & -\cos s & 0 \\
0 & 0 & 1
\end{array}\right)\left(\begin{array}{l}
x_{\alpha t s} \\
y_{\alpha t s} \\
z_{\alpha t s}
\end{array}\right)=\boldsymbol{R}_{s} \boldsymbol{x} .
$$

Now, we back substitute (5b) into (4) and (3) in turn, yielding: $\boldsymbol{X}=\boldsymbol{R}_{\alpha} \boldsymbol{R}_{\boldsymbol{t}} \boldsymbol{R}_{\boldsymbol{s}} \boldsymbol{x}=\boldsymbol{R} \boldsymbol{x}$. Because the rotation matrix $\boldsymbol{M}$ is about the mapping of coordinates from the XYZ-system to the xyz-system, we transform $\boldsymbol{X}=\boldsymbol{R} \boldsymbol{x}$ into $\boldsymbol{x}=\boldsymbol{R}^{-1} \boldsymbol{X}$, of which $\boldsymbol{R}^{-1}$ is the inverse matrix of $\boldsymbol{R} . \boldsymbol{R}$ is orthogonal, thus $\boldsymbol{R}^{-1}$ equals the transposed matrix $\boldsymbol{R}^{\boldsymbol{T}}$, by calculation, which can be represented as

$$
\boldsymbol{M}=\boldsymbol{R}^{T}=\left[\begin{array}{lll}
m_{11} & m_{12} & m_{13} \\
m_{21} & m_{22} & m_{23} \\
m_{31} & m_{32} & m_{33}
\end{array}\right]
$$

where 


\section{Dai and $L u$}

$$
\begin{aligned}
& m_{11}=-\cos \alpha \cos s-\sin \alpha \cos t \sin s, \\
& m_{12}=\sin \alpha \cos s-\cos \alpha \cos t \sin s, \\
& m_{13}=-\sin t \sin s, \\
& m_{21}=\cos \alpha \sin s-\sin \alpha \cos t \cos s, \\
& m_{22}=-\sin \alpha \sin s-\cos \alpha \cos t \cos s, \\
& m_{23}=-\sin t \cos s, \\
& m_{31}=-\sin \alpha \sin t, \\
& m_{32}=-\cos \alpha \sin t, \\
& m_{33}=\cos t .
\end{aligned}
$$

\subsection{Camera Orientations Quantification}

In practice, it's difficult to directly measure the three orientations of the camera station. As far as the operation feasibility is concerned, the coordinates of the camera location and some predefined points are much easier to quantify and obtain for engineering application. The predefined points with known object coordinates are called control points. With sufficient control points, not only the three parameters $\left(X_{o}, Y_{o}, Z_{o}\right)$ about the camera spatial location can be determined, but also three camera orientations can be inferred. According to (2), at least three control points are required to work out the total six parameters. However, when dealing with random photography, determination of the values of the parameters involves the non-linear, iterative calculation which may lead to a divergent solution (Dewitt 1996). To overcome this difficulty, the direct linear transformation (DLT) method, originally proposed by Abdel-Aziz and Karara (1971), is used to simplify the Collinearity Equations to a linear form by re-arranging and combining the terms of the equations, that is

$$
\begin{aligned}
& L_{1} X_{n}+L_{2} Y_{n}+L_{3} Z_{n}+L_{4}-x_{n}\left(L_{9} X_{n}+L_{10} Y_{n}+L_{11} Z_{n}+1\right)=0, \\
& L_{5} X_{n}+L_{6} Y_{n}+L_{7} Z_{n}+L_{8}-y_{n}\left(L_{9} X_{n}+L_{10} Y_{n}+L_{11} Z_{n}+1\right)=0 .
\end{aligned}
$$

The DLT parameters $L_{l}, \ldots, L_{11}$ are in relation to the camera parameters $\left(X_{o}, Y_{o}, Z_{o}, \alpha, t, s\right)$ and $\left(c, x_{o}, y_{o}\right)$. Nevertheless, at the cost of simplicity, the quantity of the initial DLT parameters increases to eleven. This leads to at least six control points required in solving the equations.

At a construction site, it's commonplace to utilize some surveying tools (i.e. total station, theodolite) to check the distance between the known and target locations, and measure the horizontal and vertical angles from the known to those target locations. Post processing the surveying data can conveniently produce the object positions in terms of three-dimensional coordinates.

In order to devise a pragmatic way of determining the camera station orientations, the present research attempts to use two control point locations, namely, the camera station location and the object focus location. The camera station location refers to the original point of the image coordinate system which is localized on the center of the camera image plane. Ideally, it's the point that is focused at the image plane when projecting rays of light at infinity through the perspective center. This point can be approximated as the camera perspective center. The object focus location is the point on the surface of the target object at which the camera is aimed through the center autofocus point in the viewfinder when taking photos. If there are nine autofocus points spreading on the screen of the camera viewfinder, the autofocus needs to be set to the center point of the viewfinder. Figure 6 illustrates the camera station location and the object focus location in the object coordinate system which are referred to as $P_{o}\left(X_{o}, Y_{o}, Z_{o}\right)$ and $P_{f}\left(X_{f}, Y_{f}, Z_{f}\right)$ respectively. Since the x, y axes of the image coordinate system lie on the image plane of the camera, the point $P_{o}$ and $P_{f}$ forming a line along the z-axis is perpendicular to the image plane of the camera. Hence, we can specify a vector to represent this line, that is

$$
\boldsymbol{N}=\left[\begin{array}{c}
X_{f}-X_{o} \\
Y_{f}-Y_{o} \\
Z_{f}-Z_{o}
\end{array}\right]
$$

$N$ aligns along the z-axis of the image coordinate system. Actually, it is the normal vector of the camera image plane which can be expressed in terms of $\alpha$ and $t$ orientations. 


\section{Dai and $L u$}

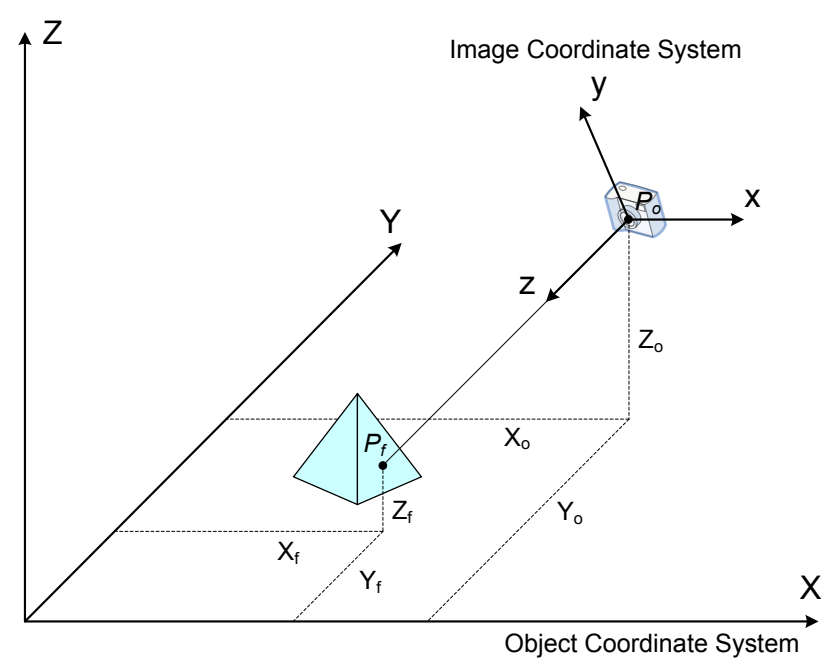

Figure 6: The camera station location and the object focus location in the object coordinate system

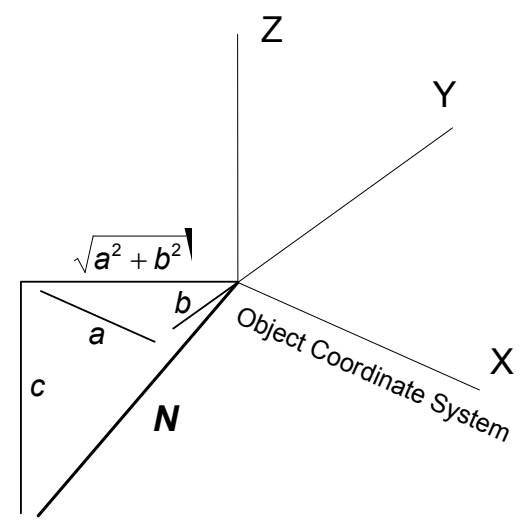

Figure 7: The normal vector $N$ at the origin of the object coordinate system

Now we use $N$ to derive the camera station orientations $(\alpha, t)$. The ranges of values of three orientations are set as

$$
\begin{aligned}
& \alpha \in\left(-180^{\circ}, 180^{\circ}\right], \\
& t \in\left[0^{\circ}, 180^{\circ}\right], \\
& s \in\left(-180^{\circ}, 180^{\circ}\right] .
\end{aligned}
$$

This not only ensures the unique correspondence relationship for all possible angular orientations between the two sets of three-dimensional coordinate systems, but also avoids the duality problem in solving triangular equations (Shih 1990). To determine $\alpha$ and $t$, Figure 7 is plotted to illustrate the normal vector $N$ at the origin of the object coordinate system, where symbols of $a, b$, and $c$ are denoted by

$$
\begin{aligned}
& a=X_{f}-X_{o}, \\
& b=Y_{f}-Y_{o}, \\
& c=Z_{f}-Z_{o} .
\end{aligned}
$$

From Figure 7, $t$ is determined by calculating the elevation angle of $\boldsymbol{N}$ with respect to the XY-plane. The formula is 


\section{Dai and $L u$}

$$
t=90^{\circ}-\arctan \left(\frac{c}{\sqrt{a^{2}+b^{2}}}\right) .
$$

$\alpha$ is determined by projecting $N$ onto the XY-plane and calculating the angle of the projection with respect to the Y-axis. The formula is

$$
\alpha=\arctan \left(\frac{a}{b}\right)
$$

As for (11), attention should be paid to the proper quadrant selection of the angle, which can be determined by the sign of the numerator $a$ and denominator $b$. A minus (-) needs to be assigned before $a$ and $b$ when performing the quadrant determination.

However, it's not sufficient only by use of this normal vector to compute the third angular orientation $s$. We further narrow the photographing condition to taking either landscape photos or portrait photos. This means the camera is always positioned parallel to the ground, thus, one of the $\mathrm{x}$ and $\mathrm{y}$ axes in image coordinate system always parallels with surface formed by $\mathrm{X}$ and $\mathrm{Y}$ axes in the object coordinate system. As such, the angular parameter $s$ of the camera rotation around the line of sight is only equal to either $180^{\circ}$ (landscape position) or $-90^{\circ}$ (counter-clockwise portrait position), $90^{\circ}$ (clockwise portrait position). It should be recognized that this is a limitation for this method. However, when applying this method in conjunction with construction applications, the requirement of the camera station's alignment to ground is practically acceptable, and the reduction of the number of control point locations considerably lowers the efforts for calibrating the camera's position and orientation at the real construction site.

\section{CASE STUDY}

To demonstrate the feasibility of this analytical approach, one case was implemented based on the site photos and the Koden test result data from a real construction site located in Hong Kong. This section discusses the production of a 3D as-built model of a bored pile and the analytical computation for immersing this 3D as-built bored pile model onto the site photo.

As a common practice at a construction site in Hong Kong, pile conditions in terms of integrity, depth and verticality need be inspected when the pile hole has been excavated and grouted. This ensures that the pile capacity is strong enough to transfer applied load from superstructure safely to the surrounding soil / rock. In this case, the Koden test was employed to measure the shaft and bellout profile, dimensions and verticality of the bored pile prior to the installation of reinforcement cage and the placement of concrete. The testing instrument was a Ultrasonic Earth Echo Auger Monitoring System Model DM-684. Figure 8 shows the raw format of the test result from Koden DM-684. In Figure 8, both the graphical profiles along $\mathrm{XX}^{\prime}$-axis and $\mathrm{YY}^{\prime}$ '-axis are plotted. Interpretation and calculation by residential engineers was instrumental in transforming the raw images into computer-aided design (CAD) drawings annotated with geometric parameters (Figure 9). From this drawing, we found an inclination below Level L occurred for this pile excavation. The inclination ratio was $1 / 58$, which exceeded the threshold value $1 / 300$ as per technical specifications. This indicated extra investigation would be required to determine whether this pile excavation would be repaired or abandoned. 


\section{Dai and $L u$}

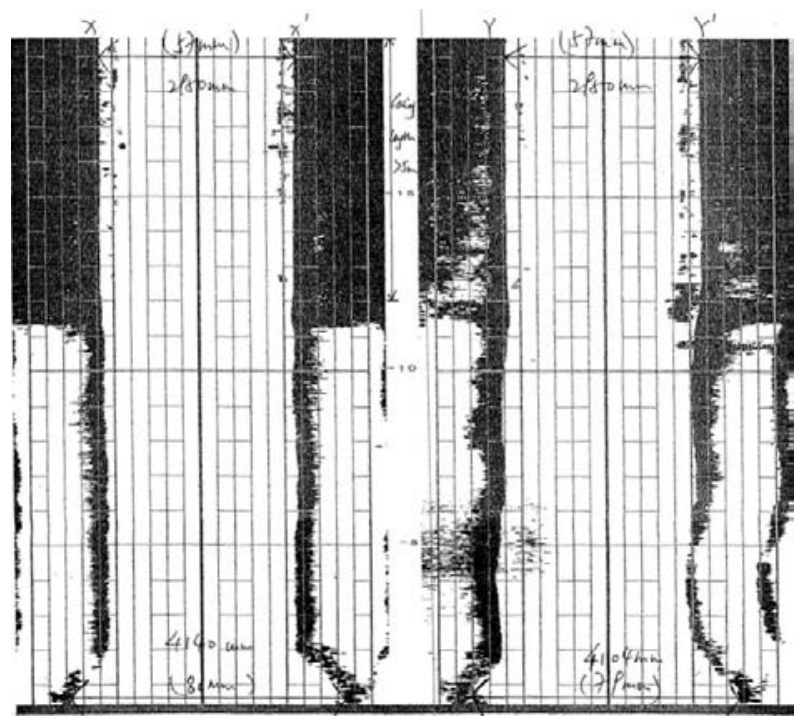

Figure 8: Test result from Koden DM-684

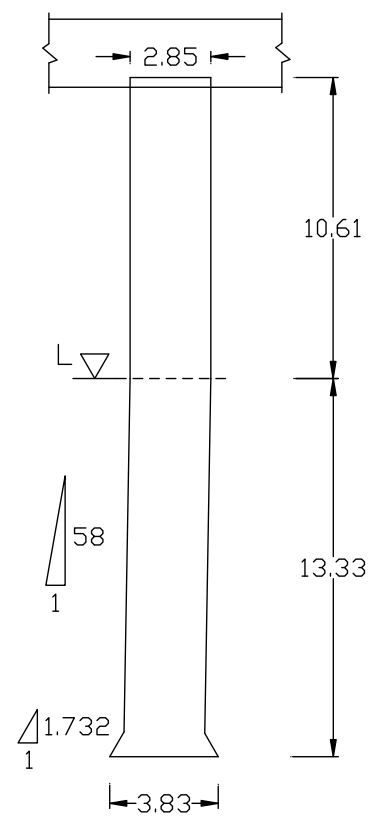

Figure 9: The computer-aided design drawing of the bored pile annotated with geometric parameters

In reality, the underground infrastructure cannot be directly photographed by camera due to the lack of line of sight. In this case, we simulate this action by using a virtual camera to photograph the $3 \mathrm{D}$ as-built model of the bored pile so as to generate a virtual photo of this bored pile. Based on the CAD drawing, we applied 3ds Max ${ }^{\circledR}$ (Autodesk Inc. 2009) to quickly model the 3D as-built bored pile. We then set up a virtual camera in the $3 \mathrm{ds}$ Max ${ }^{\circledR}$ environment with the camera setting as: landscape option $s=180^{\circ}$, camera station location $P_{o}(-18,-7.5,5)$. Note that an object coordinate system is assumed, of which the origin is at top center of the bored pile and axis unit is meter. The object focus location was selected at the fringe of the bored pile, i.e. $P_{f}(-1.425,0,0)$. Figure 10 shows the virtual camera photographing the $3 \mathrm{D}$ as-built model of the bored pile in the $3 \mathrm{ds}$ Max $^{\circledR}$ environment. 


\section{Dai and $L u$}

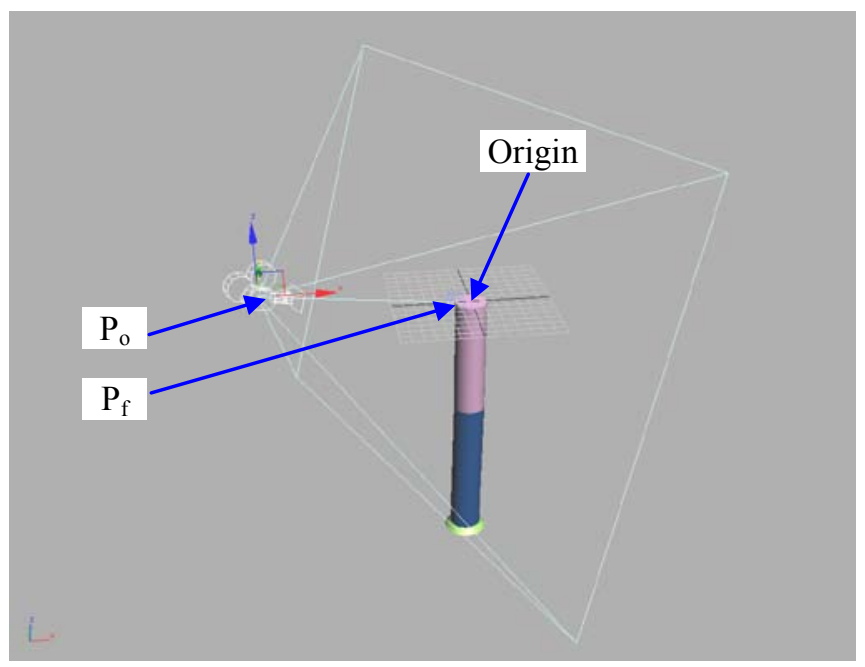

Figure 10: The virtual camera photographing the 3D as-built model of the bored pile in $3 \mathrm{ds} \operatorname{Max}^{\circledR}$ environment

By (10) and (11), we calculated the angular orientations of the virtual camera as $\left(\alpha=-114.35^{\circ}, t=105.37^{\circ}, s=180^{\circ}\right)$. Substituting these orientations in (6b), we have the rotation matrix

$$
\boldsymbol{M}=\left[\begin{array}{ccc}
-0.412249 & 0.911071 & 0.000000 \\
0.241440 & 0.109249 & 0.964247 \\
0.878497 & 0.397510 & -0.265007
\end{array}\right] .
$$

Substituting the values of elements $m_{i j}$ into (2), the location of any known spatial point on the image plane can be determined given the specified camera focal length. For validation, the object focus location point $(-1.425,0,0)$ and the determined $\boldsymbol{M}$ were entered into (2), yielding the image coordinates $(0,0)$, which means the point lying on the line of sight of the camera was projected onto the center of the image plane. This proved the correctness of the matrix. The focal length of the virtual camera was set to $14 \mathrm{~mm}$ to obtain a wide-angle photographic view. By doing so, the whole range of the pile model can be covered by the virtual camera. Last, we merged the virtual photo into the site photo. Figure 11 illustrates the augmented site photo of the as-built bored pile, on which the concreting work was just finished and the subsurface part of the bored pile was directly seen in the photo. Note in this augmented photo, the inclination below Level L for this pile is highlighted in blue. 


\section{Dai and $L u$}

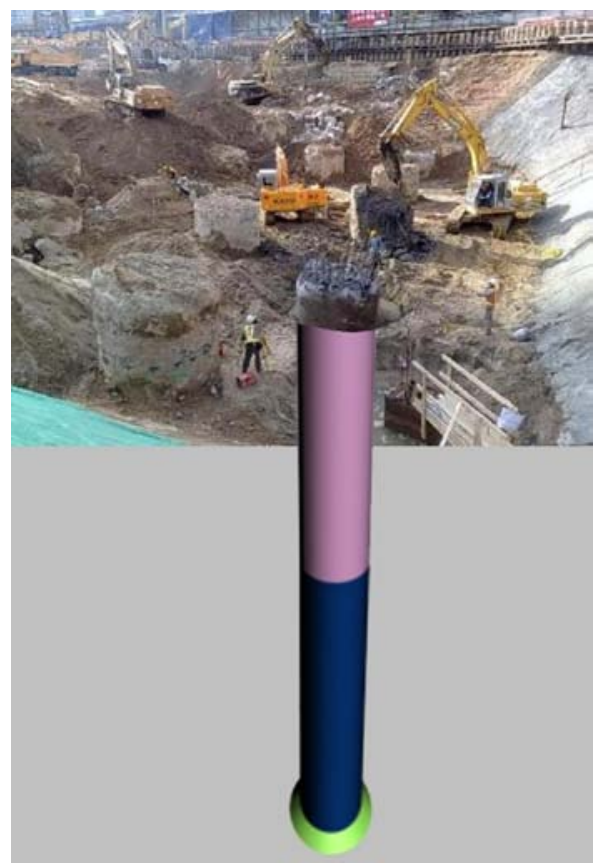

Figure 11: The augmented site photo showing both ground and underground situation of the bored pile

\section{CONCLUSIONS}

This paper firstly briefs the Koden test method which is taken as a common practice in Hong Kong to outline the profile of bored piles. Then, this paper makes an attempt at establishing photo-augmentation equations by which the 3D as-built models of bored piles can be analytically immersed onto the 2D site photos. The case of augmenting a ground site photo with as-built underground data for the purpose of investigation of alignment for a bored pile construction is demonstrated. In short, the analytical approach proposed is computationally simple and effective. It holds great application potential for visualizing the "invisible" as-built building product in an augmented site photos.

\section{ACKNOWLEDGMENTS}

This presented research was substantially funded by Hong Kong Research Grants Council (RGC) through Project PolyU 5245/08E. The writers are also grateful to Stephen Cheng Kin Kei who works with Ove Arup Hong Kong Ltd. as Residential Engineer for valuable input to this research.

\section{REFERENCES}

Abdel-Aziz, Y. I., and H. M. Karara. 1971. Direct Linear Transformation from Comparator Coordinates into Object Space Coordinates in Close-Range Photogrammetry. Proceedings of the ASP/UI Symposium on Close-Range Photogrammetry, 1-18. Univ. of Illinois, Urbana, IL.

Abeid, J. N., and D. Arditi. 2002a. Linking time-lapse digital photography and dynamic scheduling of construction operations. Journal of Computing in Civil Engineering 16 (4): 269-279. Reston, VA: ASCE.

Abeid, J. N., and D. Arditi. 2002b. Time-lapse digital photography applied to project management. Journal of Construction Engineering and Management 128(6): 530-535. Reston, VA: ASCE,.

Autodesk Inc. 2009. 3ds Max: Production-proven 3D modeling, animation, and rendering solution for entertainment and engineering application. Available via $<$ http://usa.autodesk.com/adsk/servlet/pc/index?id=135674 10 \&siteID=123112> [accessed March 2, 2009].

Dai, F., and M. Lu. 2008. Photo-Based 3D Modeling of Construction Resources for Visualization of Operations Simulation: Case of Modeling a Precast Façade. In Proceedings of the 2008 Winter Simulation Conference, ed. S. J. Mason, R. Hill, L. Moench, and O. Rose, 2439-2446. Miami, Florida, USA: Institute of Electrical and Electronics Engineers, Inc. 


\section{Dai and $L u$}

Dewitt, B. A. 1996. Initial Approximations for the Three-Dimensional Conformal Coordinate Transformation. Journal of Photogrammetric Engineering and Remote Sensing 62(1): 79-83. Bethesda, MD: American Society for Photogrammetry and Remote Sensing.

Jeong, H. S., and D. M. Abraham. 2004. A decision tool for the selection of imaging technologies to detect underground infrastructure. Tunneling and Underground Space Technology 19(2): 175-191. New York, NY: Elsevier Science.

Kim, H., and N. Kano. 2008. Comparison of construction photograph and VR image in construction progress. Automation in Construction 17(2): 137-143. New York, NY: Elsevier Science.

Luhmann, T., and W. Tecklenburg. 2001. Hybride Photogrammetric and Geodetic Surveillance of Historical Buildings for an Urban Tunnel Construction. International Workshop on Recreating the Past Visualization and Animation of Cultural Heritage, Ayuttaya, Thailand.

McGlone, J. C. 1989. Analytic Data-Reduction Schemes in Non-Topographic Photogrammetry. In Non-Topographic Photogrammetry, 2nd ed., ed. H. M. Karara, Chap. 4, 37-55. Falls Church, VA: American Society for Photogrammetry and Remote Sensing.

Shih, T. Y. 1990. The Duality and Critical Condition in the Formation and Decomposition of a Rotation Matrix. Journal of Photogrammetric Engineering and Remote Sensing 56(8): 1173-1179. Bethesda, MD: American Society for Photogrammetry and Remote Sensing.

Waugh, L. 2006. Construction Site Photography: Virtual Reality vs. The Focus+Context Problem. Proceeding of 2006 Annual CSCE Conference of the Canadian Society for Civil Engineering, Page No: CT-092 1-8, Calgary, Canada.

Wolf, P. 1983. Elements of Photogrammetry, 559-601. New York: McGraw-Hill.

Wong, K. W. 1980. Basic Mathematics of Photogrammetry. In Manual of Photogrammetry, 4th ed., ed. C. C. Slama, Chap. 2, 37-101. Falls Church, VA: American Society for Photogrammetry and Remote Sensing.

\section{AUTHOR BIOGRAPHIES}

FEI DAI received his Bachelor degree of Civil Engineering from Hefei University of Technology in 2002, Master degree of Civil Engineering from Tsinghua University in 2005, and now is a PHD student of Department of Civil and Structural Engineering at the Hong Kong Polytechnic University. His research interest is construction operations planning and optimization, as well as application of the photogrammetry technique to construction visualization and quantification. His email address is $<$ dai.f@polyu.edu.hk>.

MING LU is Associate Professor of Construction Engineering \& Management of the Department of Civil and Structural Engineering, the Hong Kong Polytechnic University. He received a Ph.D. degree from the University of Alberta, Canada. His research interest is development of easy-to-apply, cost-effective methodologies of artificial intelligence and computer simulations for construction engineering and project management. Dr Lu proposed the Simplified Discrete-Event Simulation Approach (SDESA) for construction simulation and has led the research effort of developing the SDESA computer platform. His email address is $\langle$ cemluepolyu . edu. $\mathrm{hk}\rangle$. 